\title{
Pulse wave velocity, carotid intima media thickness and flow-mediated dilation in Takayasu arteritis: a systematic review
}

Jun Watanabe ${ }^{1}$, Yoshitaka Iwazu², Kazuhiko Kotani ${ }^{1}$

\author{
${ }^{1}$ Division of Community and Family Medicine, Jichi Medical University, \\ Shimotsuke-city, Japan \\ 2Division of Anti-aging Medicine, Center for Molecular Medicine, Jichi medical \\ University, Shimotsuke-city, Japan
}

Submitted: 13 February 2021

Accepted: 16 March 2021

Arch Med Sci Atheroscler Dis 2021; 6: e79-e84

DOI: https://doi.org/10.5114/amsad.2021.105390

Copyright (c) 2021 Termedia \& Banach

\section{Abstract}

Introduction: Takayasu arteritis (TA) is a chronic vasculitis associated with an increased cardiovascular risk. The measurement of pulse wave velocity (PWV), carotid artery intima-media thickness (CIMT) and flow-mediated dilatation (FMD) are generally used for evaluating the cardiovascular risk. The application of these measurements to TA patients remains undetermined.

Material and methods: Clinical studies that reported the PWV, CIMT and FMD levels in TA patients, which were published prior to 2021, were summarized using PubMed.

Results: Fifteen studies were eligible. Overall, in TA patients, the PWV and CIMT levels were significantly higher and the FMD levels were significantly lower compared to controls. Part of the studies showed that the disease activity of TA was significantly associated with the PWV, CIMT or FMD levels. Conclusions: The PWV, CIMT and FMD measurements could be useful for evaluating the cardiovascular risk in TA patients. Further studies to determine the proper use of these measurements are warranted.

Key words: cardiovascular disease, endothelial dysfunction, pulse wave analysis, Takayasu disease, vascular stiffness.

\section{Introduction}

Takayasu arteritis (TA), which is also known as pulseless disease and aortitis syndrome, is a chronic granulomatous vasculitis affecting large- and medium-sized arteries, predominantly the aorta and its main branches [1-3]. The aorta can be involved along its entire length, and although any of its branches can be diseased, the subclavian and common carotid arteries are most commonly affected [1]. This disease is comparatively rare, typically presenting before the age of 40 years with a female predominance [1-3]. Although the etiology of TA is unknown, it is assumed to have an autoimmune basis, influenced by genetic and environmental factors [2, 4]. TA is seen worldwide, but its incidence is higher in Southeast Asia, Central and South America, and Africa [4]. The distribution of vascular lesions can differ among regions; that is, cervical and thoracic arterial lesions are prevalent in Japan and South America, whereas abdominal lesions involving the renal artery are prevalent in Israel and other Asian countries [5-7].

\author{
Corresponding author: \\ Prof. Kazuhiko Kotani \\ Division of Community \\ and Family Medicine \\ Jichi Medical University \\ 3311-1 Yakushiji \\ 329-0498 Shimotsuke-city \\ Japan \\ Phone: +81285 587394 \\ E-mail: kazukotani@jichi.ac.jp
}


The prognosis of TA has improved but is often affected by vascular complications [8-12]. Atherosclerotic plaques are common in lesions where the primary vessel disease was prominent and local lesions occur in TA patients $[13,14]$. In TA patients, cardiovascular events are considered the main causes of mortality $[1,3,15,16]$. In this context, a link between local and systemic atherosclerosis is a known phenomenon [17, 18].

There are some surrogate markers of future cardiovascular events. The measurement of pulse wave velocity (PWV) is a simple and non-invasive test to evaluate arterial stiffness, an atherosclerotic manifestation [19, 20]. High-resolution B-mode ultrasound measurements of the carotid artery intima-media thickness (CIMT) are a direct and non-invasive index to evaluate atherosclerosis [21]. Flow-mediated dilatation (FMD) of the brachial artery is an ultrasound method that is widely used to evaluate the endothelial function [22]. Endothelial dysfunction is on the atherogenic pathway, and reduces the levels of FMD [22-24].

Given the at-risk cardiovascular events in TA patients, it would be useful to know whether the PWV, CIMT and FMD levels are out-of-normal range and then whether these measurements can be used to evaluate the degree of risk. The present study aimed to review the available literature on the application of these measurements in TA patients.

\section{Literature search}

The PubMed database was searched for clinical studies published prior to January 2021. If needed, a manual search was performed according to the citation lists of the relevant literature. The following key words were used: ("Takayasu arteritis"[Mesh] OR "Takayasu arteritis"[tiab] OR "pulseless disease"[tiab] OR "aortitis syndrome" [tiab]), which were combined with ("pulse wave analysis"[Mesh] OR "Vascular Stiffness"[Mesh] OR "Carotid Intima-Media Thickness"[Mesh] OR "Carotid Intimal Medial Thickness 1"[Supplementary Concept] OR "pulse wave"[tiab] OR "Vascular stiffness"[tiab] OR “intima media”[tiab] OR “IMT”[tiab] OR "flow mediated dilation"[tiab] OR "endothelial dysfunction"[tiab]). The studies were restricted to those written in the English language. The list of titles and abstracts was initially screened for relevance by the two review authors (JW and YI). We then selected original articles that were full-length publications in peer-review journals. In addition, the reference lists of selected articles were also manually reviewed. Any disagreements were resolved by discussion, or by involving a third review author (KK). Articles were excluded if any of the following criteria were present: (1) did not compare patients to a healthy control group; (2) the article was based on animal studies; (3) the article was based on in vitro or experimental model-based studies; or (4) the article was a review.

\section{Results}

The results of the literature search are shown in Figure 1. We retrieved 40 articles from the initial search. Of these, 26 articles were excluded after judgment of their eligibility according to screening of titles and abstracts. Two articles were requoted from the reference lists of eligibly identified studies. A total of 15 studies were available for the present review [13, 25-38].

\section{Pulse wave velocity}

As shown in Table I, there were six studies that evaluated the PWV levels [25-30], two studies used both carotid-radial PWV (crPWV) and carotid-femoral PWV (cfPWV) [25, 26], two studies used brachial-ankle PWV (baPWV) [27, 28], and two studies used cfPWV [29, 30]. The systolic blood pressure (BP) levels [25-27, 30] and diastolic BP levels $[26,27]$ were significantly higher in TA patients than in controls. In two other studies, the pulse pressure (PA) levels in TA patients were higher than those in controls, although the systolic and diastolic BP levels were similar in TA patients and controls $[28,29]$.

The baPWV and cfPWV levels were significantly higher in TA patients than in controls in all the studies [25-30]. One study on crPWV showed that the TA patients in patients were higher than those in controls [26], while another did not [25].

In addition, the levels of diastolic BP [25], mean BP [29] and body mass index [25] were positively associated with PWV. The PWV levels were not correlated with inflammatory markers, including the erythrocyte sedimentation rate (ESR) [25, 28-30] and C-reactive protein (CRP) [25, 27-30]. A study reported no association between the PWV levels and disease activity, the interval from the onset of TA or the cumulative dosage of glucocorticoid agents [29]. A study showed that the baPWV levels in TA patients with coronary artery disease were associated with the severity of coronary artery disease and major adverse cardiac events [27].

\section{Intima-media thickness}

Eight studies evaluated the CIMT levels (Table II) $[13,31-37]$. All of the studies reported that the CIMT levels in TA patients were significantly higher than those in controls [13, 31-37].

Four studies investigated the association between the CIMT levels and carotid artery lesions (CALs) $[13,32,34,36]$. Three of the four studies showed that the CIMT levels were significantly higher in TA patients with CALs in comparison to 


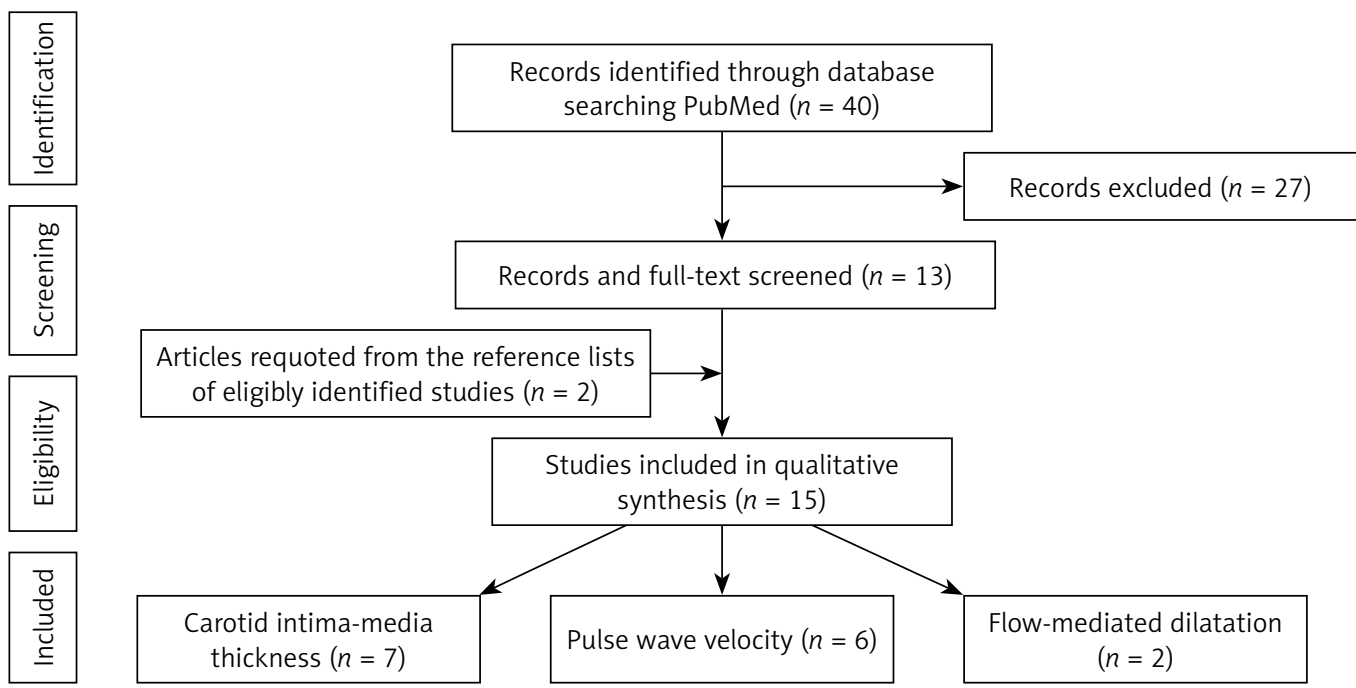

Figure 1. Flowchart of the selection of study articles

Table I. PWV in patients with TA

\begin{tabular}{|c|c|c|c|c|c|c|c|c|c|}
\hline \multirow{2}{*}{$\begin{array}{l}\text { PWV } \\
\text { type }\end{array}$} & \multirow{2}{*}{$\begin{array}{l}\text { Authors } \\
\text { (ref) }\end{array}$} & \multirow[t]{2}{*}{ Year } & \multicolumn{2}{|c|}{$n$ (male) } & \multicolumn{2}{|c|}{ Age [years] } & \multirow{2}{*}{$\begin{array}{l}\text { Interval } \\
\text { from the } \\
\text { onset } \\
\text { [years] }\end{array}$} & \multicolumn{2}{|c|}{$\mathrm{PWV}[\mathrm{cm} / \mathrm{s}]$} \\
\hline & & & TA & Control & TA & Control & & TA & Control \\
\hline \multirow{2}{*}{$\begin{array}{l}\text { Carotid- } \\
\text { radial } \\
\text { PWV }\end{array}$} & $\mathrm{Ng}[25]$ & 2006 & $10(0)$ & $11(0)$ & $41.0 \pm 12.5$ & $32.4 \pm 5.5$ & 2 to 20 & $1040 \pm 280$ & $1090 \pm 140$ \\
\hline & $\begin{array}{c}\text { Grotenhuis } \\
{[26]}\end{array}$ & 2019 & $7(1)$ & $7(1)$ & $13.8 \pm 3.2$ & $13.8 \pm 3.0$ & $2.5 \pm 3.8$ & $8100 \pm 1800^{*}$ & $6400 \pm 600$ \\
\hline \multirow{2}{*}{$\begin{array}{l}\text { Brachio- } \\
\text { ankle } \\
\text { PWV }\end{array}$} & Wang [27] & 2015 & $48(12)$ & $40(11)$ & $45.0 \pm 6.7$ & $44.5 \pm 5.6$ & n.r. & $1700 \pm 380^{*}$ & $1380 \pm 300$ \\
\hline & Wang [28] & 2020 & $67(0)$ & $67(0)$ & $35.68 \pm 10.42$ & $39.67 \pm 9.29$ & n.r. & $1496 \pm 432^{*}$ & $1211 \pm 154$ \\
\hline \multirow{4}{*}{$\begin{array}{l}\text { Carotid- } \\
\text { femoral } \\
\text { PWV }\end{array}$} & $\mathrm{Ng}$ [25] & 2006 & $10(0)$ & $11(0)$ & $41.0 \pm 12.5$ & $32.4 \pm 5.5$ & 2 to $>20$ & $1200 \pm 430^{*}$ & $830 \pm 110$ \\
\hline & Neto [29] & 2014 & $27(0)$ & $27(0)$ & $32.37 \pm 8.26$ & $33.89 \pm 10.12$ & $10 \pm 7.2$ & $977 \pm 349^{*}$ & $783 \pm 106$ \\
\hline & Yang [30] & 2017 & $25(0)$ & $25(0)$ & $28.3 \pm 6.2$ & $27.1 \pm 4.2$ & $1.2 \pm 0.7$ & $837 \pm 223^{*}$ & $646 \pm 115$ \\
\hline & $\begin{array}{c}\text { Grotenhuis } \\
{[26]}\end{array}$ & 2019 & $7(1)$ & $7(1)$ & $13.8 \pm 3.2$ & $13.8 \pm 3.0$ & $2.5 \pm 3.8$ & $8300 \pm 1900^{*}$ & $5100 \pm 800$ \\
\hline
\end{tabular}

PWV - pulse wave velocity, n.r. - not reported, TA - Takayasu arteritis. * ${ }^{*}$-value $<0.05$.

Table II. CIMT in patients with TA

\begin{tabular}{|c|c|c|c|c|c|c|c|c|}
\hline \multirow{2}{*}{$\begin{array}{l}\text { Authors } \\
\text { (Ref) }\end{array}$} & \multirow[t]{2}{*}{ Year } & \multicolumn{2}{|c|}{$n$ (male) } & \multicolumn{2}{|c|}{ Age [years] } & \multirow{2}{*}{$\begin{array}{c}\text { Interval from } \\
\text { the onset } \\
\text { [years] }\end{array}$} & \multicolumn{2}{|c|}{ Mean CIMT [mm] } \\
\hline & & TA & Control & TA & Control & & TA & Control \\
\hline $\begin{array}{l}\text { Raninen } \\
{[31]}\end{array}$ & 1996 & $16(2)$ & $16(2)$ & 45.7 & 46.4 & 16.4 & $\begin{array}{l}\text { Rt: } 1.2 \pm 0.5^{*} \\
\text { Lt: } 1.8 \pm 0.9^{*}\end{array}$ & $\begin{array}{l}\text { Rt: } 0.7 \pm 0.1 \\
\text { Lt: } 0.7 \pm 0.1\end{array}$ \\
\hline $\begin{array}{l}\text { Taniguchi } \\
\text { [32] }\end{array}$ & 1997 & $22(2)$ & $19(0)$ & 41.2 & 41.2 & 10.3 & $1.6 \pm 0.6^{\star}$ & $0.7 \pm 0.1$ \\
\hline $\begin{array}{l}\text { Zieliński } \\
\text { [33] }\end{array}$ & 2002 & $18(3)$ & 17 (n.r.) & 38.6 & n.r. & 7.7 & $1.1 \pm 0.1^{\star}$ & $0.59 \pm 0.01$ \\
\hline Seth [34] & 2006 & $37(12)$ & 28 (n.r.) & $26.1 \pm 5.6$ & n.r. & n.r. & $1.3 \pm 0.1^{\star}$ & $0.6 \pm 0.1$ \\
\hline $\begin{array}{l}\text { Seyahi } \\
\text { [13] }\end{array}$ & 2006 & $30(0)$ & $50(0)$ & $35.4 \pm 8.0$ & $38.2 \pm 5.7$ & 8.8 & $1.0 \pm 0.3^{\star}$ & $0.6 \pm 0.08$ \\
\hline $\begin{array}{l}\text { Bicakcigil } \\
\text { [35] }\end{array}$ & 2011 & $28(4)$ & $98(6)$ & $36.8 \pm 10$ & $38.7 \pm 6.6$ & n.r. & $0.6 \pm 0.1^{*}$ & $0.5 \pm 0.01$ \\
\hline $\begin{array}{l}\text { Alibaz- } \\
\text { Oner [36] }\end{array}$ & 2014 & $32(2)$ & $20(3)$ & $39.06 \pm 11.44$ & $36.7 \pm 11.9$ & 5.9 & $1.1 \pm 0.3^{\star}$ & $0.7 \pm 0.08$ \\
\hline $\begin{array}{l}\text { Svensson } \\
{[37]}\end{array}$ & 2020 & $25(4)$ & $25(4)$ & $23(11-71)$ & $46(23-64)$ & 4.5 & $1.3 \pm 0.8^{\star}$ & $0.5 \pm 0.04$ \\
\hline
\end{tabular}

CIMT - carotid artery intima-media thickness, Lt - left, n.r. - not reported, Rt - right, TA - Takayasu arteritis. ${ }^{*} p$-value $<0.05$. 
TA patients without CALs [13, 32, 34]. The CIMT levels were positively correlated with the severity of luminal stenosis in TA patients [32, 34]. In angiographically normal carotid arteries, the CIMT levels were significantly higher in TA patients [32] and clinically active TA patients [34] in comparison to controls [34]. In contrast, another study showed no correlation between the CIMT levels and the presence of CALs [36].

Two studies showed that the CIMT levels were positively associated with disease activity [34, 37], although another study showed that CIMT levels were not associated with the disease activity or the interval from the onset of TA [36].

\section{Flow-mediated dilation}

Two studies evaluated the FMD levels (Table III) $[36,38]$. These studies reported that the FMD levels in TA patients were significantly lower than those in controls [36, 38]. Two studies reported no correlation between the FMD levels and disease duration [36, 38] and disease activity [36]. A study showed that glucocorticoid agents had no effect on the FMD levels [36]. Another study showed that TA patients receiving prednisone treatment had significantly lower FMD levels in comparison to non-patient controls and patients who did not receive prednisone [38].

\section{Discussion}

The present review revealed that in TA patients, overall, the PWV and CIMT levels were higher, and the FMD levels were lower than in controls. This confirms that TA patients have accelerated atherosclerotic conditions. In order to evaluate the cardiovascular risk, the PWV, CIMT and FMD measurements are suggested to be applicable in clinical practice in TA patients.

Although we did not identify any well-designed prospective cohort studies that investigated the cardiovascular outcomes among the studies included in the present review, TA patients with serious complications and/or a progressive disease course have been previously indicated to exhibit a lower survival rate [8]. Because the levels of
CIMT and FMD, in addition to PWV, generally predict cardiovascular outcomes [19-24], according to the general consensus, it is thought that these values can be reasonably applied in clinical practice to predict an increased cardiovascular risk in TA patients.

A link between local and systemic atherosclerosis is of interest $[17,18]$. In the studies of PWV that were included in this review, the PWV levels were not correlated with inflammatory markers $[25,27-30]$ or the disease activity or duration (interval from the onset) of TA [29]. However, two studies reported that the PWV and total vascular wall thicknesses were prominent in the carotid, subclavian, and aortic arteries, which are predominantly affected by TA, rather than in the peripheral brachial, radial, and femoral arteries, which are infrequently affected by the disease [25, 31]. In this review, while a study reported that the CIMT levels are not associated with the disease activity of TA [36], two studies showed that the CIMT levels were positively associated with the disease activity of TA $[34,37]$. The carotid plaques were present on the side of CALs in TA patients with CALs [13]. The CIMT levels were associated with the severity of CAD [32, 34]. In this review, there were no associations between the FMD levels and the disease activity or the duration (interval from the onset) of TA $[36,38]$. Accordingly, part of the studies likely support the presence of a link between local and systemic atherosclerosis $[17,18]$. The findings also appear to show a weak influence of the cumulative burden on the localized artery (as expressed by the disease activity or duration) on systemic atherosclerotic conditions, which may be modified by the beneficial effect of treatments on vascular pathophysiology.

Steroids, glucocorticoid agents, which are routinely used in the treatment of TA, can suppress immunological reactions and inflammation but are also pro-atherogenic, leading to weight gain, dyslipidemia, insulin resistance and sodium retention, resulting in hypertension $[39,40]$. Three studies included in this review reported no correlation between the cumulative dosage of glucocorticoids and the PWV levels [29] or the presence of

Table III. FMD in patients with TA

\begin{tabular}{|c|c|c|c|c|c|c|c|c|}
\hline \multirow{2}{*}{$\begin{array}{l}\text { Authors } \\
\text { (Ref) }\end{array}$} & \multirow[t]{2}{*}{ Year } & \multicolumn{2}{|c|}{$n$ (male) } & \multicolumn{2}{|c|}{ Age [years] } & \multirow{2}{*}{$\begin{array}{l}\text { Interval } \\
\text { from the } \\
\text { onset } \\
\text { [years] }\end{array}$} & \multicolumn{2}{|c|}{ FMD (\%) } \\
\hline & & TA & Control & TA & Control & & TA & Control \\
\hline $\begin{array}{l}\text { Espinola- } \\
\text { Zavaleta } \\
{[38]}\end{array}$ & 2009 & $30(0)$ & $30(0)$ & $36.5 \pm 11.8$ & $35.6 \pm 12.5$ & 10.9 & $7.7^{\star}$ & 15.1 \\
\hline $\begin{array}{l}\text { Alibaz- } \\
\text { Oner } \\
{[36]}\end{array}$ & 2014 & $32(2)$ & $20(3)$ & $39.1 \pm 11.4$ & $36.7 \pm 11.9$ & 5.9 & $7.6 \pm 4.7^{*}$ & $15.6 \pm 2.2$ \\
\hline
\end{tabular}

FMD - flow-mediated dilatation, TA - Takayasu arteritis. ${ }^{*} p$-value $<0.05$ 
plaques [13], and no effect of the glucocorticoid dosage on the FMD levels [36]. Even though the results in the population of TA patients combined with controls revealed that glucocorticoids could decrease the FMD levels [38], it seemed that glucocorticoid agents did not strongly accelerate atherosclerosis.

Two studies of PWV included in this review reported that the diastolic BP [25] and mean BP [29], but not the systolic BP [25], affected cfPWV. In addition, a study in this review reported that the systolic BP was a predictor of FMD [38]. Therefore, these results suggested that BP was strongly associated with PWV and FMD. Hypertension is a key cardiovascular complication [41] and is reported in $33-83 \%$ of TA patients, generally reflecting renal artery stenosis, which are seen in $28-75 \%$ of patients [42]. Hypertension can also develop from aortic stiffness [41]. Comorbid hypertension was previously reported to be a possible determinant of long-term cardiovascular outcomes in TA patients [8, 10, 27]. These findings suggested that the increased BP might be a therapeutic target for improving the cardiovascular risk in TA patients.

The present review was associated with several limitations. All of the included studies analyzed relatively small numbers of patients. The use of diagnostic criteria (i.e., the American College of Rheumatology criteria [43]) was not fully unified across the studies [30, 33, 34]. The information regarding the age and sex of controls $[33,34]$ and the influence of treatment for TA was not fully described (with the exception of two studies [29, 36]). Although the PWV levels are typically regulated by BP [44, 45], a BP-adjusted analysis was not performed in all the studies [25-30]. None of the studies investigated the change in PWV, CIMT and FMD over time during the follow-up period. These points should be addressed in future investigations.

\section{Perspectives}

The PWV, CIMT and FMD measurements would be suitable for the simple and repeated evaluation of the cardiovascular risk in TA patients. However, PWV (a marker of arterial stiffness), CIMT (a structural change of the arterial wall) and FMD (a marker of endothelial dysfunction) can have similar meanings with some differences in the prediction of the cardiovascular risk [19, 21, 22]. We have not yet described the proper use of the respective measurements because we found that there were no studies that simultaneously examined the PWV, CIMT and FMD levels in TA patients in the present review. Further studies should be performed to determine whether or not there is an appropriate choice of measurements. Furthermore, the strategies of monitoring and therapeutic interventions using these measurements are still unestablished. Thus, prospective, longitudinal and outcome observations and interventional studies are needed.

The present review partly supports the hypothesis that local vessel wall lesions can affect systemic atherosclerosis in TA [15, 16, 25, 31, 32, 34, 37]. The local biomolecules that are distributed to blood flow following shear stress of abnormal arteries may play a persistent role in the development of systemic cardiovascular events [46]. Mechanistic studies should be undertaken to investigate the relationship to PWV, CIMT and FMD measurements in TA.

In conclusion, TA patients showed high PWV and CIMT levels, and low FMD levels in comparison to controls. The PWV, CIMT and FMD measurements could be useful to evaluate the cardiovascular risk in TA patients. Future studies are warranted.

\section{Conflict of interest}

The authors declare no conflict of interest.

\section{References}

1. Mason JC. Takayasu arteritis-advances in diagnosis and management. Nat Rev Rheumatol 2010; 6: 406-15.

2. Wen D, Du X, Ma CS. Takayasu arteritis: diagnosis, treatment and prognosis. Int Rev Immunol 2012; 31: 462-73.

3. Koster MJ, Matteson EL, Warrington KJ. Recent advances in the clinical management of giant cell arteritis and Takayasu arteritis. Curr Opin Rheumatol 2016; 28: 211-7.

4. Vaideeswar P, Deshpande JR. Pathology of Takayasu arteritis: a brief review. Ann Pediatr Cardiol 2013; 6: 52-8.

5. Numano F. Differences in clinical presentation and outcome in different countries for Takayasu's arteritis. Curr Opin Rheumatol 1997; 9: 12-5.

6. Isobe M. Takayasu arteritis revisited: current diagnosis and treatment. Int J Cardiol 2013; 168: 3-10.

7. Trinidad B, Surmachevska N, Lala V. Takayasu Arteritis. StatPearls [Internet], Treasure Island (FL) 2020.

8. Ishikawa K, Maetani S. Long-term outcome for 120 Japanese patients with Takayasu's disease. Clinical and statistical analyses of related prognostic factors. Circulation 1994; 90: 1855-60.

9. Numano F, Okawara M, Inomata H, Kobayashi Y. Takayasu's arteritis. Lancet 2000; 356: 1023-5.

10. Tervaert JW. Translational mini-review series on immunology of vascular disease: accelerated atherosclerosis in vasculitis. Clin Exp Immunol 2009; 156: 377-85.

11. Ohigashi H, Haraguchi G, Konishi M, et al. Improved prognosis of Takayasu arteritis over the past decade comprehensive analysis of 106 patients. Circ J 2012; 76: 1004-11.

12. Willem J, Tervaert C. Cardiovascular disease due to accelerated atherosclerosis in systemic vasculitides. Best Pract Res Clin Rheumatol 2013; 27: 33-44.

13. Seyahi E, Ugurlu S, Cumali R, et al. Atherosclerosis in Takayasu arteritis. Ann Rheum Dis 2006; 65: 1202-7.

14. Seyahi E, Ucgul A, Cebi Olgun D, et al. Aortic and coronary calcifications in Takayasu arteritis. Semin Arthritis Rheum 2013; 43: 96-104.

15. Ishikawa K. Survival and morbidity after diagnosis of occlusive thromboaortopathy (Takayasu's disease). Am J Cardiol 1981; 47: 1026-32. 
16. Maksimowicz-McKinnon K, Clark TM, Hoffman GS Limitations of therapy and a guarded prognosis in an American cohort of Takayasu arteritis patients. Arthritis Rheum 2007; 56: 1000-9.

17. Willerson JT. Systemic and local inflammation in patients with unstable atherosclerotic plaques. Prog Cardiovasc Dis 2002; 44: 469-78.

18. Herrero-Fernandez B, Gomez-Bris R, Somovilla-Crespo B, et al. Immunobiology of atherosclerosis: a complex net of interactions. Int J Mol Sci 2019; 20: 5293.

19. European Network for Non-invasive Investigation of Large Arteries. Expert consensus document on arterial stiffness: methodological issues and clinical applications. Eur Heart J 2006; 27: 2588-605.

20. Jain S, Khera R, Corrales-Medina VF, et al. Inflammation and arterial stiffness in humans. Atherosclerosis 2014 237: 381-90.

21. de Groot E, Hovingh GK, Wiegman A, et al. Measurement of arterial wall thickness as a surrogate marker for atherosclerosis. Circulation 2004; 109: III33-8.

22. Charakida M, Masi S, Lüscher TF, et al. Assessment of atherosclerosis: the role of flow-mediated dilatation Eur Heart J 2010; 31: 2854-61.

23. Heitzer T, Schlinzig T, Krohn K, et al. Endothelial dysfunction, oxidative stress, and risk of cardiovascular events in patients with coronary artery disease. Circulation 2001; 104: 2673-8.

24. Taddei S, Salvetti A. Endothelial dysfunction in essential hypertension: clinical implications. J Hypertens 2002; 20: 1671-4.

25. Ng WF, Fantin F, Ng C, et al. Takayasu's arteritis: a cause of prolonged arterial stiffness. Rheumatology 2006; 45: 741-5.

26. Grotenhuis HB, Aeschlimann FA, Hui W, et al. Increased arterial stiffness adversely affects left ventricular mechanics in patients with pediatric Takayasu arteritis from a Toronto cohort. J Clin Rheumatol 2019; 25: 171-5.

27. Wang X, Dang A. Prognostic value of brachial-ankle pulse wave velocity in patients with Takayasu arteritis with drug-eluting stent implantation. Arthritis Care Res 2015; 67: 1150-7.

28. Wang Z, Dang A, Lv N. Brachial-ankle pulse wave velocity is increased and associated with disease activity in patients with Takayasu arteritis. J Atheroscler Thromb 2020; 27: 172-82

29. Neto NSR, Levy-Neto M, Tolezani EC, et al. Determinants of arterial stiffness in female patients with Takayasu arteritis. J Rheumatol 2014; 41: 1374-8.

30. Yang Y, Wang Z, Yuan LJ, et al. Aortic stiffness evaluated by echocardiography in female patients with Takayasu's arteritis. Clin Exp Rheumatol 2017; 35: 134-8.

31. Raninen RO, Kupari MM, Pamilo MS, et al. Arterial wall thickness measurements by B mode ultrasonography in patients with Takayasu's arteritis. Ann Rheum Dis 1996; 55: 461-5.

32. Taniguchi N, Itoh K, Honda M, et al. Comparative ultrasonographic and angiographic study of carotid arterial lesions in Takayasu's arteritis. Angiology 1997; 48: 9-20.

33. Zieliński T, Wolkanin-Bartnik J, Mekowiecka-Cicsla, et al. Ultrasound examination of carotid arteries with intima media measurement: an underestimated tool in the diagnosis of Takayasu's disease. Int J Angiol 2002; 11: 153-7.

34. Seth S, Goyal NK, Jagia P, et al. Carotid intima-medial thickness as a marker of disease activity in Takayasu's arteritis. Int J Cardiol 2006; 108: 385-90.
35. Bicakcigil M, Tasan D, Tasdelen N, et al. Role of fibrinolytic parameters and plasminogen activator inhibitor 1 (PAI-1) promoter polymorphism on premature atherosclerosis in SLE patients. Lupus 2011; 20: 1063-71.

36. Alibaz-Oner F, Yurdakul S, Aytekin S, et al. Impaired endothelial function in patients with Takayasu's arteritis. Acta Cardiol 2014; 69: 45-9.

37. Svensson C, Eriksson P, Zachrisson H. Vascular ultrasound for monitoring of inflammatory activity in Takayasu arteritis. Clin Physiol Funct Imaging 2020; 40: 37-45.

38. Espinola-Zavaleta N, Soto-López ME, Carreón-Torres E, et al. Altered flow-mediated vasodilatation, low paraoxonase- 1 activity, and abnormal high-density lipoprotein subclass distribution in Takayasu's arteritis. Circ J 2009 73: 760-6.

39. Verlato F, Salmistraro G, Avruscio GP, et al. Takayasu's arteritis: anatomic change before and after steroid therapy evaluated by angiography and echo-Doppler color-flow. Int Angiol 1992; 11: 233-6.

40. Fukudome $\mathrm{Y}$, Abe I, Onaka U, et al. Regression of carotid wall thickening after corticosteroid therapy in Takayasu's arteritis evaluated by B-mode ultrasonography: report of 2 cases. J Rheumatol 1998; 25: 2029-32.

41. Chatterjee S, Flamm SD, Tan CD, et al. Clinical diagnosis and management of large vessel vasculitis: Takayasu arteritis. Curr Cardiol Rep 2014; 16: 499.

42. Johnston SL, Lock RJ, Gompels MM. Takayasu arteritis: a review. J Clin Pathol 2002; 55: 481-6.

43. Arend WP, Michel BA, Bloch DA, et al. The American College of Rheumatology 1990 criteria for the classification of Takayasu arteritis. Arthritis Rheum 1990; 33: 1129-34.

44. McEniery CM, Yasmin, Hall IR, et al. Normal vascular aging: differential effects on wave reflection and aortic pulse wave velocity: the Anglo-Cardiff Collaborative Trial (ACCT). J Am Coll Cardiol 2005; 46: 1753-60.

45. Cecelja M, Chowienczyk P. Dissociation of aortic pulse wave velocity with risk factors for cardiovascular disease other than hypertension: a systematic review. Hypertension 2009; 54: 1328-36.

46. Numano F, Kishi Y, Tanaka A, et al. Inflammation and atherosclerosis. Atherosclerotic lesions in Takayasu arteritis. Ann N Y Acad Sci 2000; 902: 65-76. 